Research Article

\title{
Pulsatile Flow of Two-Fluid Nonlinear Models for Blood Flow through Catheterized Arteries: A Comparative Study
}

\author{
D. S. Sankar ${ }^{1}$ and Usik Lee ${ }^{2}$ \\ ${ }^{1}$ School of Mathematical Sciences, Science University of Malaysia, Penang 11800, Malaysia \\ ${ }^{2}$ Department of Mechanical Engineering, Inha University, 253 Yonghyun-Dong, Nam-Gu, \\ Incheon 402-751, South Korea \\ Correspondence should be addressed to Usik Lee, ulee@inha.ac.kr
}

Received 3 September 2009; Revised 7 December 2009; Accepted 21 January 2010

Academic Editor: Ekaterina Pavlovskaia

Copyright (C) 2010 D. S. Sankar and U. Lee. This is an open access article distributed under the Creative Commons Attribution License, which permits unrestricted use, distribution, and reproduction in any medium, provided the original work is properly cited.

\begin{abstract}
The pulsatile flow of blood through catheterized arteries is analyzed by treating the blood as a two-fluid model with the suspension of all the erythrocytes in the core region as a non-Newtonian fluid and the plasma in the peripheral layer as a Newtonian fluid. The non-Newtonian fluid in the core region of the artery is represented by (i) Casson fluid and (ii) Herschel-Bulkley fluid. The expressions for the flow quantities obtained by Sankar (2008) for the two-fluid Casson model and Sankar and Lee (2008) for the two-fluid Herschel-Bulkley model are used to get the data for comparison. It is noted that the plug-flow velocity, velocity distribution, and flow rate of the twofluid H-B model are considerably higher than those of the two-fluid Casson model for a given set of values of the parameters. Further, it is found that the wall shear stress and longitudinal impedance are significantly lower for the two-fluid H-B model than those of the two-fluid Casson model.
\end{abstract}

\section{Introduction}

Catheters are of extensive use in modern medicine. In routine clinical studies, the measurement of arterial blood pressure/pressure gradient and flow velocity/flow rate are achieved by the use of an appropriate catheter tool device in the desired part of the arterial network [1]. Catheters are also used in diagnostic techniques (such as X-ray angiography, intravascular ultrasound, and coronary balloon angioplasty) as well as in the treatment (balloon angioplasty) of various arterial diseases [2]. Catheters are even used to clear the short occlusions from the walls of the stenosed artery [3]. By reducing the obstruction through balloon angioplasty, the mean translesional pressure drop is reduced [4] and the coronary blood flow as well as the coronary flow reserve is increased [5]. The insertion 
of a catheter in an artery will alter the flow field, modify the pressure distribution, and hence increases the flow resistance [6]. Thus, the pressure or pressure gradient recorded by a transducer attached to the catheter will differ from that of an uncatheterized artery, and hence it is essential to know the catheter-induced error [7]. Even, a very small angioplasty guidewire leads to a sizable increase in flow resistance [8]. For smaller infusion catheter, the increase in flow resistance is less, although still appreciable. Hence, it is meaningful to study the increase in flow resistance due to catheterization.

Several theoretical and experimental investigations are performed to study the dynamics of blood flow through catheterized arteries [9-14]. MacDonald [15] discussed the blood flow characteristics in catheterized arteries using conformal transformation and finite difference method. Sarkar and Jayaraman [1] obtained the correction to flow ratepressure drop relationship in coronary angioplasty with steady steaming effect. Dash et al. [6] analyzed the effect of catheterization on various flow characteristics in a curved artery using perturbation method. Dash and Daripa [16] have studied the blood flow characteristics in an eccentric catheterized artery using a fast algorithm. In all the above investigations, Newtonian fluid represents blood. But it is well known that blood, being suspension of cells, behaves like a nonNewtonian fluid at low shear rate $(\dot{\gamma}<10 / \mathrm{sec})$ and during its flow through narrow blood vessels of diameter 0.02-0.1 mm [17-19]. Dash et al. [3] studied the steady and pulsatile flow of Casson fluid for blood flow through catheterized arteries and estimated the increase in frictional resistance using the perturbation analysis. Sankar and Hemalatha $[7,20]$ have studied the steady and pulsatile flow Herschel-Bulkley fluid for blood flow through catheterized arteries using perturbation method and estimated the increase in longitudinal impedance to flow.

Srivastava and Saxena [19] and Misra and Pandey [21] have mentioned that for blood flowing through narrow blood vessels there is a peripheral layer of plasma and a core region of suspension of all the erythrocytes. Hence, for a more realistic description of blood flow, it is appropriate to treat the blood as a two-fluid model consisting of a core region containing all the erythrocytes as a nonNewtonian fluid and the plasma in the peripheral layer as a Newtonian fluid [19-21]. Sankar and Lee [22] and Sankar [23] have analyzed the pulsatile flow of two-phase fluid models for blood flow through catheterized narrow arteries at low shear rates, by treating the fluid in the core region as Herschel-Bulkley (H-B) model and (ii) Casson model, respectively. In both of the two-fluid models, Newtonian fluid represents the fluid in the peripheral layer.

It is noticed that blood obeys Casson's equation only for moderate shear rate and the Herschel-Bulkley equation represents fairly closely what is occurring in blood $[7,18]$. Chaturani and Ponnalagar Samy [24] have mentioned that for tube diameter $0.095 \mathrm{~mm}$ blood behaves like H-B fluid rather than power-law and Bingham fluids. Iida [25] reports "That velocity profile in the arterioles having diameter less than $0.1 \mathrm{~mm}$ are generally explained fairly by the two models. However, velocity profiles in the arterioles whose diameters are less than $0.065 \mathrm{~mm}$ does not conform to the Casson model but can still be explained by H-B fluid model. Moreover, H-B fluid model can be reduced to power-law fluid model when the yield stress is zero and Bingham fluid model when its power-law index $n$ takes the value 1, so that the two-fluid power-law and Bingham models can be studied from the two-fluid H-B model itself as its particular cases. Thus, the two-fluid H-B model has more suitability than the two-fluid Casson model in the studies of blood flow through narrow arteries. Hence, in this paper, the expressions for the flow quantities obtained by Sankar and Lee [22] for the two-fluid H-B model and the expressions for the flow quantities obtained by Sankar [23] for the two-fluid Casson model are used to compare these fluid models and bring out the 
advantage of using the two-fluid H-B model over two-fluid Casson model for blood flow in catheterized arteries. In this study, the governing equations and the boundary conditions of both of the two-fluid models and the expressions obtained for the various flow quantities of these models by Sankar and Lee [22] and Sankar [23] are mentioned in brief and are used to perform a comparative study. The layout of the paper is as follows.

The formulation and method of solution of (i) two-phase Casson fluid model and (ii) two-phase Herschel-Bulkley (H-B) fluid model are briefly given in Section 2. The variations of the flow quantities of these two-fluid models on the yield stress, catheter radius ratio and pulsatility of the flow are analyzed in Section 3. The increase in the longitudinal impedance to flow due to catheterization for different types of catheters which are used in clinics, is also computed for both of the two-phase fluid models and is discussed in Section 3. The results are summarized in the concluding Section 4 .

\section{Mathematical Formulation}

Consider an axially symmetric, laminar, pulsatile, and fully developed unidirectional flow of blood (assumed to be incompressible) in the axial direction in an artery in which a catheter is introduced coaxially, where the artery is modeled as a rigid-walled circular tube of radius

$\bar{R}$. The catheter radius is taken to be $k \bar{R}(k<1)$. Blood is represented by a two-fluid model with the suspension of all of the erythrocytes in the core region as a nonNewtonian fluid and the plasma in the peripheral region as a Newtonian fluid. The nonNewtonian fluid in the core region is represented by (i) Casson fluid model and (ii) Herschel-Bulkley fluid model. We have used the cylindrical polar coordinates $(\bar{r}, \bar{\phi}, \bar{z})$, where $\bar{r}$ and $\bar{z}$ denote the radial and axial coordinates and $\bar{\phi}$ is the azimuthal angle. The flow geometries of the two-fluid model for blood flow through catheterized artery are shown in Figure 1.

\subsection{Two-Fluid Casson Model}

\subsubsection{Governing Equations and Boundary Conditions}

For unidirectional flow of blood (incompressible fluid) in the axial direction, the equation of continuity reduces to

$$
\left(\frac{\partial \bar{\rho}}{\partial \bar{t}}+\bar{u}_{r} \frac{\partial \bar{u}_{z}}{\partial \bar{r}}+\frac{\bar{u}_{\phi}}{\bar{r}} \frac{\partial \bar{u}_{z}}{\partial \bar{\phi}}+\bar{u}_{z} \frac{\partial \bar{u}_{z}}{\partial \bar{z}}\right)=0 .
$$

Since the flow is axisymmetric and one dimensional in the axial direction, the radial component of the velocity $\bar{u}_{r}$ and the circumferential component of the velocity $\bar{u}_{\phi}$ do not exit (become zero) and only the axial component of the velocity $\bar{u}_{z}$ exists, and hence, the equation of continuity (2.1a) reduces to

$$
\frac{\partial \bar{u}_{z}}{\partial \bar{z}}=0
$$




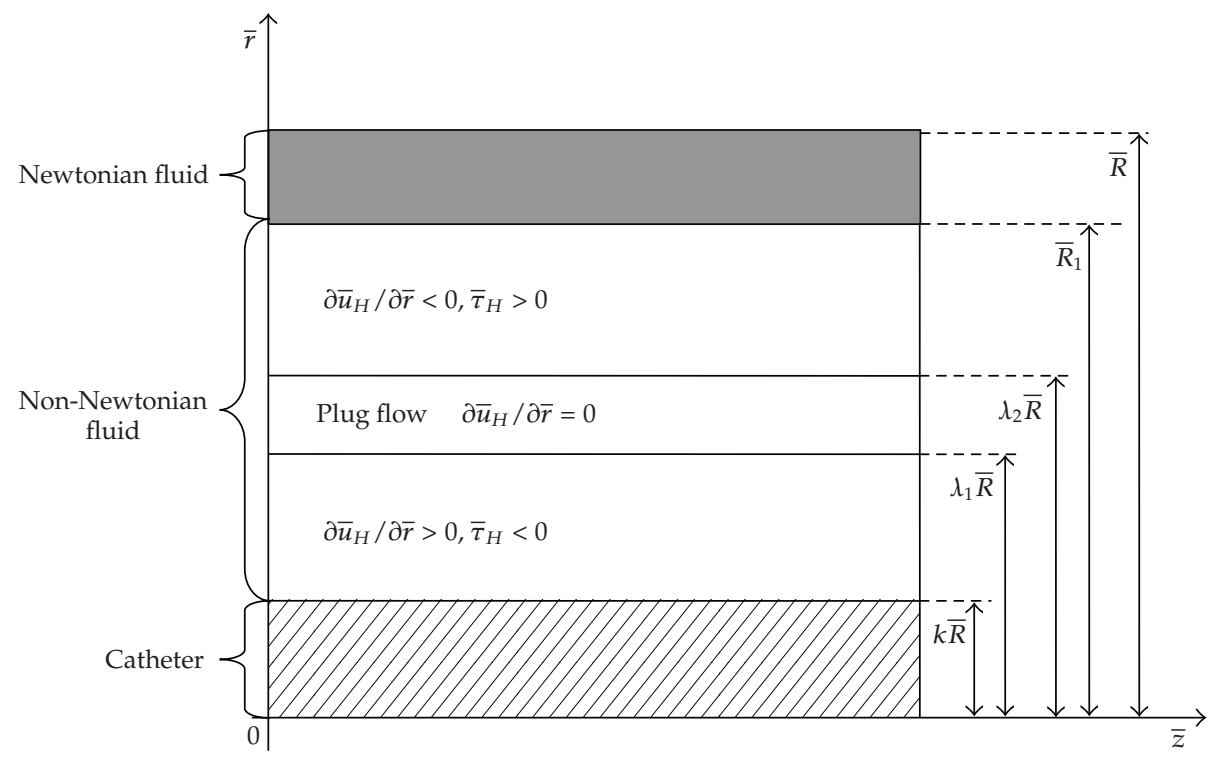

Figure 1: Geometry of the catheterized artery modeled as two-fluid model. The flowing blood in the artery is modeled as a two-fluid model with the fluid (suspension of all the erythrocytes) in the core region being treated as nonNewtonian fluid and the fluid (plasma) in the peripheral layer region being treated as Newtonian fluid. Catheter is inserted into the artery coaxially. Since, the nonNewtonian fluid that we assume in the core region of the two-fluid model has yield stress, there is a plug-flow region which is bounded by the yield planes.

The axial component of the momentum equation for the one-dimensional flow of blood in the axial direction is given as

$$
\bar{\rho}\left(\frac{\partial \bar{u}_{z}}{\partial \bar{t}}+\bar{u}_{r} \frac{\partial \bar{u}}{\partial \bar{r}}+\frac{\bar{u}_{\phi}}{r} \frac{\partial \bar{u}_{z}}{\partial \bar{\phi}}+\bar{u}_{z} \frac{\partial \bar{u}_{z}}{\partial \bar{z}}\right)=-\frac{\partial \bar{p}}{\partial \bar{z}}-\frac{1}{\bar{r}} \frac{\partial}{\partial \bar{r}}(\overline{r \tau})
$$

Using (2.1b) and $\bar{u}_{r}=\bar{u}_{\phi}=0$ (the radial component of the velocity $\bar{u}_{r}$ and circumferential component of the velocity $\bar{u}_{\phi}$ become zero for one-dimensional flow of blood in the axial direction) in the momentum equation (2.2a), we get

$$
\bar{\rho} \frac{\partial \bar{u}_{z}}{\partial \bar{t}}=-\frac{\partial \bar{p}}{\partial \bar{z}}-\frac{1}{\bar{r}} \frac{\partial}{\partial \bar{r}}(\bar{r} \bar{\tau})
$$

The momentum equation (2.2b) is rewritten for the fluid flow in the core region and peripheral region of a two-fluid model, respectively, as

$$
\begin{array}{ll}
\bar{\rho}_{C} \frac{\partial \bar{u}_{C}}{\partial \bar{t}}=-\frac{\partial \bar{p}}{\partial \bar{z}}-\frac{1}{\bar{r}} \frac{\partial}{\partial \bar{r}}\left(\bar{r} \bar{\tau}_{C}\right) \quad \text { if } k \bar{R} \leq \bar{r} \leq \bar{R}_{1}, \\
\bar{\rho}_{N} \frac{\partial \bar{u}_{N}}{\partial \bar{t}}=-\frac{\partial \bar{p}}{\partial \bar{z}}-\frac{1}{\bar{r}} \frac{\partial}{\partial \bar{r}}\left(\bar{r} \bar{\tau}_{N}\right) \quad \text { if } \bar{R}_{1} \leq \bar{r} \leq \bar{R},
\end{array}
$$


where $\bar{p}$ denotes the pressure; $\bar{\rho}_{C}$ and $\bar{\rho}_{N}$ denote the density of the Casson fluid and Newtonian fluid, respectively; $\bar{\tau}_{C}$ and $\bar{\tau}_{N}$ denote the shear stress of the Casson fluid and Newtonian fluid, respectively; $\bar{u}_{C}$ and $\bar{u}_{N}$ denote the fluid's velocity in the core region and peripheral region, respectively; $\bar{t}$ denotes the time; $\bar{R}_{1}$ is the radius of the core region of the artery. The simplified form of the constitutive equations of the fluid in motion in the core region (Casson fluid) and peripheral layer (Newtonian fluid) are given by

$$
\begin{aligned}
& \bar{\mu}_{C} \frac{\partial \bar{u}_{C}}{\partial \bar{r}}=\left|\bar{\tau}_{C}\right|\left[1-\frac{2 \sqrt{\bar{\tau}_{y}}}{\sqrt{\left|\bar{\tau}_{C}\right|}}+\frac{\bar{\tau}_{y}}{\left|\bar{\tau}_{C}\right|}\right] \quad \text { if } \frac{\partial \bar{u}_{C}}{\partial \bar{r}}>0, \bar{\tau}_{C}<0, k \bar{R} \leq \bar{r} \leq \lambda_{1} \bar{R}, \\
& \bar{\mu}_{C} \frac{\partial \bar{u}_{C}}{\partial \bar{r}}=-\left|\bar{\tau}_{C}\right|\left[1-\frac{2 \sqrt{\bar{\tau}_{y}}}{\sqrt{\left|\bar{\tau}_{C}\right|}}+\frac{\bar{\tau}_{y}}{\left|\bar{\tau}_{C}\right|}\right] \quad \text { if } \frac{\partial \bar{u}_{C}}{\partial \bar{r}}<0, \bar{\tau}_{C}>0, \lambda_{2} \bar{R} \leq \bar{r} \leq \bar{R}_{1} \text {, } \\
& \frac{\partial \bar{u}_{C}}{\partial \bar{r}}=0 \quad \text { if }\left|\bar{\tau}_{C}\right| \leq \bar{\tau}_{y}, \lambda_{1} \bar{R} \leq \bar{r} \leq \lambda_{2} \bar{R}, \\
& \bar{\mu}_{N} \frac{\partial \bar{u}_{N}}{\partial \bar{r}}=-\left|\bar{\tau}_{N}\right| \quad \text { if } \frac{\partial \bar{u}_{N}}{\partial \bar{r}}<0, \bar{\tau}_{N}>0, \bar{R}_{1} \leq \bar{r} \leq \bar{R},
\end{aligned}
$$

where $\bar{\mu}_{C}$ and $\bar{\mu}_{N}$ are the viscosities of the Casson fluid and Newtonian fluid, respectively; $\bar{\tau}_{y}$ is the yield stress; $\lambda 1$ and $\lambda 2$ are the yield planes bounding the plug-flow region. Equations (2.3a), (2.3b), and (2.6) are equipped with the following boundary conditions:

$$
\begin{gathered}
\frac{\partial \bar{u}_{C}}{\partial \bar{r}}=0 \quad \text { at } \bar{r}=0, \quad \bar{u}_{N}=0 \quad \text { at } r=\bar{R}, \\
\bar{u}_{C}=\bar{u}_{N}, \quad \bar{\tau}_{C}=\bar{\tau}_{N} \quad \text { at } \bar{r}=\bar{R}_{1} \text { (at the interface). }
\end{gathered}
$$

\subsubsection{Nondimensionalization}

Let $\bar{p}_{0}$ be the absolute magnitude of the typical pressure gradient. Let us introduce the following nondimensional variables:

$$
\begin{gathered}
u_{C}=\frac{\bar{u}_{C}}{\left(\bar{p}_{0} \bar{R}^{2} / 2 \bar{\mu}_{C}\right)}, \quad u_{N}=\frac{\bar{u}_{N}}{\left(\bar{p}_{0} \bar{R}^{2} / 2 \bar{\mu}_{N}\right)}, \quad r=\frac{\bar{r}}{\bar{R}}, \quad R_{1}=\frac{\bar{R}_{1}}{\bar{R}}, \\
z=\frac{\bar{z}}{\bar{R}}, \quad \tau_{C}=\frac{\bar{\tau}_{C}}{\left(\bar{p}_{0} \bar{R} / 2\right)}, \quad \tau_{N}=\frac{\bar{\tau}_{N}}{\left(\bar{p}_{0} \bar{R} / 2\right)}, \quad \theta=\frac{\bar{\tau}_{y}}{\left(\bar{p}_{0} \bar{R} / 2\right)}, \\
t=\bar{\omega} \bar{t}, \quad \varepsilon_{C}=\alpha_{C}^{2}=\frac{\bar{R}_{0}^{2} \bar{\rho}_{C} \bar{\omega}}{\bar{\mu}_{C}}, \quad \varepsilon_{N}=\alpha_{N}^{2}=\frac{\bar{R}_{0}^{2} \bar{\rho}_{N} \bar{\omega}}{\bar{\mu}_{N}}
\end{gathered}
$$


where $\alpha_{C}$ and $\alpha_{N}$ are the Womersley numbers of the Casson fluid and Newtonian fluid, respectively, and $\theta$ is the nondimensional yield stress. The pressure gradient can be written as

$$
\frac{\partial \bar{p}}{\partial \bar{z}}(\bar{t})=-\bar{p}_{0} P(t)
$$

where $P(t)$ is the nondimensional pressure gradient along the axis, which is taken as a periodic function of time for pulsatile flow. Using (2.8) and (2.9), the momentum equations (2.3a) and (2.3b), and the constitutive equations (2.4)-(2.6) are simplified, respectively, to

$$
\begin{gathered}
\varepsilon_{C} \frac{\partial u_{C}}{\partial t}=2 P(t)-\frac{1}{r} \frac{\partial}{\partial r}\left(r \tau_{C}\right) \quad \text { if } k \leq r \leq R_{1}, \\
\varepsilon_{N} \frac{\partial u_{N}}{\partial t}=2 P(t)-\frac{1}{r} \frac{\partial}{\partial r}\left(r \tau_{N}\right) \quad \text { if } R_{1} \leq r \leq 1, \\
\frac{\partial u_{C}}{\partial r}=\left|\tau_{C}\right|\left(1-\frac{2 \sqrt{\theta}}{\left|\sqrt{\tau_{C}}\right|}+\frac{\theta}{\left|\tau_{C}\right|}\right) \quad \text { if } \frac{\partial u_{C}}{\partial r}>0, \tau_{C}<0, k \leq r \leq \lambda_{1}, \\
\frac{\partial u_{C}}{\partial r}=-\left|\tau_{C}\right|\left(1-\frac{2 \sqrt{\theta}}{\left|\sqrt{\tau_{C}}\right|}+\frac{\theta}{\left|\tau_{C}\right|}\right) \quad \text { if } \frac{\partial u_{C}}{\partial r}<0, \tau_{C}>0, \lambda_{2} \leq r \leq R_{1}, \\
\frac{\partial u_{C}}{\partial r}=0 \quad \text { if }\left|\tau_{C}\right| \leq \theta, \lambda_{1} \leq r \leq \lambda_{2}, \\
\frac{\partial u_{N}}{\partial r}=-\left|\tau_{N}\right| \quad \text { if } \frac{\partial u_{N}}{\partial r}<0, \tau_{N}>0, R_{1} \leq r \leq 1 .
\end{gathered}
$$

The boundary conditions (in the nondimensional form) are

$$
\begin{gathered}
\frac{\partial u_{C}}{\partial r}=0 \quad \text { at } r=0, \quad u_{N}=0 \quad \text { at } r=1, \\
u_{C}=u_{N}, \quad \tau_{C}=\tau_{N} \quad \text { at } r=R_{1} \text { (at the interface). }
\end{gathered}
$$

\subsubsection{Perturbation Method}

Since it is not possible to find an exact solution of the nonlinear coupled implicit system of partial differential equations (2.10)-(2.15), the perturbation method is used to solve the system of nonlinear partial differential equations. When we nondimensionalize (2.3a), the Womersley number $\alpha_{C}$ occurs naturally, and hence; it is appropriate to expand the unknown $u_{C}$ in powers of $\varepsilon_{C}=\alpha_{C}^{2}$ as below:

$$
u_{C}(r, t)=u_{0 C}(r, t)+\varepsilon_{C} u_{1 C}(r, t)+\cdots .
$$


Similarly, one can expand the other unknowns $\tau_{C}, \tau_{N}$, and $u_{N}$ in the perturbation series as in (2.17). Hereafter, for our convenience, we have used " $P$ " instead of " $P(t)$ ". Using the perturbation series expansions in the momentum equations (2.10) and (2.11) and then equating the constant terms and the first-order terms, we get

$$
\begin{aligned}
& 0=2 P-\frac{1}{r} \frac{\partial}{\partial r}\left(r \tau_{0 C}\right), \\
& \frac{\partial u_{0 C}}{\partial t}=-\frac{1}{r} \frac{\partial}{\partial r}\left(r \tau_{1 C}\right), \\
& 0=2 P-\frac{1}{r} \frac{\partial}{\partial r}\left(r \tau_{0 N}\right), \\
& \frac{\partial u_{0 N}}{\partial t}=-\frac{1}{r} \frac{\partial}{\partial r}\left(r \tau_{1 N}\right) .
\end{aligned}
$$

Using the perturbation series expansions of $u_{C}, u_{N}, \tau_{C}$, and $\tau_{N}$ in the constitutive equations (2.12) $-(2.15)$ and then equating the constant terms and the first-order terms, we obtain

When $k \leq r \leq \lambda_{1}$,

$$
\begin{gathered}
\frac{\partial u_{0 C}}{\partial r}=\left(\left|\tau_{0 C}\right|-2 \sqrt{\theta\left|\tau_{0 C}\right|}+\theta\right) \quad \text { if } \frac{\partial u_{0 C}}{\partial r}>0, \tau_{0 C}<0 \\
\frac{\partial u_{1 C}}{\partial r}=\left|\tau_{1 C}\right|\left(1-\sqrt{\frac{\theta}{\left|\tau_{0 C}\right|}}\right) \quad \text { if } \frac{\partial u_{1 C}}{\partial r}>0, \tau_{1 C}<0
\end{gathered}
$$

When $\lambda_{1} \leq r \leq \lambda_{2}$,

$$
\begin{array}{ll}
\frac{\partial u_{0 C}}{\partial r}=0 & \text { if }\left|\tau_{0 C}\right|<\theta, \\
\frac{\partial u_{1 C}}{\partial r}=0 & \text { if }\left|\tau_{1 C}\right|<\theta .
\end{array}
$$

When $\lambda_{2} \leq r \leq R_{1}$,

$$
\begin{gathered}
\frac{\partial u_{0 C}}{\partial r}=-\left(\left|\tau_{0 C}\right|-2 \sqrt{\theta\left|\tau_{0 C}\right|}+\theta\right) \quad \text { if } \frac{\partial u_{0 C}}{\partial r}<0, \tau_{0 C}>0, \\
\frac{\partial u_{1 C}}{\partial r}=-\left|\tau_{1 C}\right|\left(1-\sqrt{\frac{\theta}{\left|\tau_{0 C}\right|}}\right) \quad \text { if } \frac{\partial u_{1 C}}{\partial r}<0, \tau_{1 C}>0 .
\end{gathered}
$$


When $R_{1} \leq r \leq 1$,

$$
\begin{aligned}
& \frac{\partial u_{0 N}}{\partial r}=-\left|\tau_{0 N}\right| \text { if } \frac{\partial u_{0 N}}{\partial r}<0, \tau_{0 N}>0, R_{1} \leq r \leq 1, \\
& \frac{\partial u_{1 N}}{\partial r}=-\left|\tau_{1 N}\right| \quad \text { if } \frac{\partial u_{1 N}}{\partial r}<0, \tau_{1 N}>0, R_{1} \leq r \leq 1 .
\end{aligned}
$$

The boundary conditions (2.16) become

$$
\begin{gathered}
\frac{\partial u_{0 C}}{\partial r}=\frac{\partial u_{1 C}}{\partial r}=0 \quad \text { at } r=0, \quad u_{0 N}=u_{1 N}=0 \quad \text { at } r=1, \\
u_{0 C}=u_{0 N}, \quad u_{1 C}=u_{1 N}, \quad \tau_{O C}=\tau_{0 N}, \quad \tau_{1 C}=\tau_{1 N} \quad \text { at } r=R_{1} .
\end{gathered}
$$

Equations (2.18)-(2.23) are solved explicitly with the help of the boundary conditions (2.24). For detailed derivation of the solution to variables $u_{0 C}, u_{1 C}, u_{0 N}, u_{1 N}, \tau_{0 C}, \tau_{1 C}, \tau_{0 N}$ and $\tau_{1 N}$ from (2.18)-(2.24), one can refer Sankar [23]. The detailed derivation for the flow rate, wall shear stress and longitudinal impedance are given by Sankar [23] and one can go through this reference to know about the details of obtaining these flow quantities.

\subsection{Two-Fluid Herschel-Bulkley Model}

\subsubsection{Governing Equations and Boundary Conditions}

Following the derivation of (2.3a) and (2.3b), the basic momentum equations in this case simplify to

$$
\begin{array}{cc}
\bar{\rho}_{H} \frac{\partial \bar{u}_{H}}{\partial \bar{t}}=-\frac{\partial \bar{p}}{\partial \bar{z}}-\frac{1}{\bar{r}} \frac{\partial}{\partial \bar{r}}\left(\bar{r} \bar{\tau}_{H}\right) & \text { if } k \bar{R} \leq \bar{r} \leq \bar{R}_{1} \\
\bar{\rho}_{N} \frac{\partial \bar{u}_{N}}{\partial \bar{t}}=-\frac{\partial \bar{p}}{\partial \bar{z}}-\frac{1}{\bar{r}} \frac{\partial}{\partial \bar{r}}\left(\bar{r} \bar{\tau}_{N}\right) & \text { if } \bar{R}_{1} \leq \bar{r} \leq \bar{R}
\end{array}
$$

where $\bar{p}$ denotes the pressure; $\bar{\rho}_{H}$ and $\bar{\rho}_{N}$ denote the density of the Herschel-Bulkley (H-B) fluid and Newtonian fluid, respectively; $\bar{\tau}_{H}$ and $\bar{\tau}_{N}$ denote the shear stress of the H-B fluid and Newtonian fluid, respectively; $\bar{u}_{H}$ and $\bar{u}_{N}$ denote the fluid's velocity in the core region and peripheral region, respectively; $\bar{t}$ denotes the time; $\bar{R}_{1}$ is the radius of the core region of 
the artery. The simplified form of the constitutive equations of the fluid in motion in the core region (H-B fluid) and peripheral layer (Newtonian fluid) are given by

$$
\begin{gathered}
\bar{\mu}_{H} \frac{\partial \bar{u}_{H}}{\partial \bar{r}}=\left|\bar{\tau}_{H}\right|^{n}\left(1-\frac{n \bar{\tau}_{y}}{\left|\bar{\tau}_{H}\right|}\right) \quad \text { if } \frac{\partial \bar{u}_{H}}{\partial \bar{r}}>0, \bar{\tau}_{H}<0, k \bar{R} \leq \bar{r} \leq \lambda_{1} \bar{R}, \\
\bar{\mu}_{H} \frac{\partial \bar{u}_{H}}{\partial \bar{r}}=-\left|\bar{\tau}_{H}\right|^{n}\left(1-\frac{n \bar{\tau}_{y}}{\left|\bar{\tau}_{H}\right|}\right) \quad \text { if } \frac{\partial \bar{u}_{H}}{\partial \bar{r}}<0, \bar{\tau}_{H}>0, \lambda_{2} \bar{R} \leq \bar{r} \leq \bar{R}_{1}, \\
\frac{\partial \bar{u}_{H}}{\partial \bar{r}}=0 \quad \text { if } \bar{\tau}_{H} \leq \bar{\tau}_{y}, \lambda_{1} \bar{R} \leq \bar{r} \leq \lambda_{2} \bar{R}, \\
\bar{\mu}_{N} \frac{\partial \bar{u}_{N}}{\partial \bar{r}}=-\left|\bar{\tau}_{N}\right| \quad \text { if } \frac{\partial \bar{u}_{N}}{\partial \bar{r}}<0, \bar{\tau}_{N}>0, \bar{R}_{1} \leq \bar{r} \leq \bar{R},
\end{gathered}
$$

where $\bar{\mu}_{H}, \bar{\mu}_{N}$ are the viscosities of the H-B fluid and Newtonian fluid; $\bar{\tau}_{y}$ is the yield stress; $\lambda_{1}$ and $\lambda_{2}$ are the yield planes bounding the plug-flow region. Equations (2.25), (2.26) and (2.27)-(2.30) can be solved with the help of the following boundary conditions:

$$
\begin{gathered}
\frac{\partial \bar{u}_{H}}{\partial \bar{r}}=0 \quad \text { at } \bar{r}=0, \quad \bar{u}_{N}=0 \quad \text { at } r=\bar{R}, \\
\bar{u}_{H}=\bar{u}_{N}, \quad \bar{\tau}_{H}=\bar{\tau}_{N} \quad \text { at } \bar{r}=\bar{R}_{1} \text { (at the interface). }
\end{gathered}
$$

\subsubsection{Nondimensionalization}

Let $\bar{p}_{0}$ be the absolute magnitude of the typical pressure gradient. Let us introduce the following nondimensional variables:

$$
\begin{gathered}
u_{H}=\frac{\bar{u}_{H}}{\left(\bar{p}_{0} \bar{R}^{2} / 2 \bar{\mu}_{0}\right)}, \quad u_{N}=\frac{\bar{u}_{N}}{\left(\bar{p}_{0} \bar{R}^{2} / 2 \bar{\mu}_{N}\right)}, \quad r=\frac{\bar{r}}{\bar{R}}, \quad R_{1}=\frac{\bar{R}_{1}}{\bar{R}}, \\
z=\frac{\bar{z}}{\bar{R}}, \quad \tau_{H}=\frac{\bar{\tau}_{H}}{\left(\bar{p}_{0} \bar{R} / 2\right)}, \quad \tau_{N}=\frac{\bar{\tau}_{N}}{\left(\bar{p}_{0} \bar{R} / 2\right)}, \quad \theta=\frac{\bar{\tau}_{y}}{\left(\bar{p}_{0} \bar{R} / 2\right)}, \\
t=\bar{\omega} \bar{t}, \quad \varepsilon_{H}=\alpha_{H}^{2}=\frac{\bar{R}_{0}^{2} \bar{\rho}_{H} \bar{\omega}}{\bar{\mu}_{0}}, \quad \varepsilon_{N}=\alpha_{N}^{2}=\frac{\bar{R}_{0}^{2} \bar{\rho}_{N} \bar{\omega}}{\bar{\mu}_{N}},
\end{gathered}
$$

where $\bar{\mu}_{0}=\bar{\mu}_{H}\left(2 / \bar{p}_{0} \bar{R}\right)^{n-1}$ is the typical viscosity coefficient having the dimension as that of the Newtonian fluid's viscosity, $\alpha_{H}$ and $\alpha_{N}$ are the Womersley numbers of the H-B fluid and Newtonian fluid, respectively and $\theta$ is the nondimensional yield stress. The pressure gradient can be written as

$$
\frac{\partial \bar{p}}{\partial \bar{z}}(\bar{t})=-\bar{p}_{0} P(t),
$$


where $P(t)$ is the nondimensional pressure gradient along the $z$ axis. Using (2.32) and (2.33), the momentum equations (2.25) and (2.26) and the constitutive equations (2.27)-(2.30) are simplified, respectively, to

$$
\begin{gathered}
\varepsilon_{H} \frac{\partial u_{H}}{\partial t}=2 P(t)-\frac{1}{r} \frac{\partial}{\partial r}\left(r \tau_{H}\right) \quad \text { if } k \leq r \leq R_{1}, \\
\varepsilon_{N} \frac{\partial u_{N}}{\partial t}=2 P(t)-\frac{1}{r} \frac{\partial}{\partial r}\left(r \tau_{N}\right) \quad \text { if } R_{1} \leq r \leq 1, \\
\frac{\partial u_{H}}{\partial r}=\left|\tau_{H}\right|^{n}\left(1-\frac{n \theta}{\left|\tau_{H}\right|}\right) \quad \text { if } \frac{\partial u_{H}}{\partial r}>0, \tau_{H}<0, k \leq r \leq \lambda_{1}, \\
\frac{\partial u_{H}}{\partial r}=-\left|\tau_{H}\right|^{n}\left(1-\frac{n \theta}{\left|\tau_{H}\right|}\right) \quad \text { if } \frac{\partial u_{H}}{\partial r}<0, \tau_{H}>0, \lambda_{2} \leq r \leq R_{1}, \\
\frac{\partial u_{H}}{\partial r}=0 \quad \text { if }\left|\tau_{H}\right| \leq \theta, \lambda_{1} \leq r \leq \lambda_{2}, \\
\frac{\partial u_{N}}{\partial r}=-\left|\tau_{N}\right| \quad \text { if } \frac{\partial u_{N}}{\partial r}<0, \tau_{N}>0, R_{1} \leq r \leq 1 .
\end{gathered}
$$

The boundary conditions (in the nondimensional form) are

$$
\begin{gathered}
u_{H}=0 \quad \text { at } r=k, \quad u_{N}=0 \quad \text { at } r=1, \\
u_{H}=u_{N}, \quad \tau_{H}=\tau_{N} \quad \text { at } r=R_{1} \text { (at the interface). }
\end{gathered}
$$

\subsubsection{Perturbation Method}

As it is not possible to find an analytic solution of the nonlinear coupled implicit system of partial differential equations (2.34)-(2.39), a perturbation method is used to solve the system of partial differential equations. When we nondimensionalize (2.25) and (2.26), the Womersley numbers $\alpha_{H}$ and $\alpha_{N}$ occur naturally and hence it is appropriate to expand the unknowns $\tau_{H}, \tau_{N}, u_{H}$, and $u_{N}$ in powers of $\varepsilon_{H}=\alpha_{H}^{2}$ and $\varepsilon_{N}=\alpha_{N}^{2}$. Let us expand the velocity $u_{H}$ in the perturbation series as below:

$$
u_{H}(r, t)=u_{0 H}(r, t)+\varepsilon_{H} u_{1 H}(r, t)+\cdots .
$$

Similarly, one can expand $\tau_{H}, \tau_{N}$, and $u_{N}$ in the perturbation series as in (2.41). Hereafter, for convenience, we have used " $P$ " instead of " $P(t)$ ". Using the perturbation series expansion of $\tau_{H}, \tau_{N}, u_{H}$, and $u_{N}$ in the momentum equations (2.34) and (2.35) and the constitutive 
equations (2.36)-(2.39) and then equating the constant terms and the first-order terms, one can obtain

$$
\begin{gathered}
0=2 P(t)-\frac{1}{r} \frac{\partial}{\partial r}\left(r \tau_{0 H}\right), \\
\frac{\partial u_{0 H}}{\partial t}=-\frac{1}{r} \frac{\partial}{\partial r}\left(r \tau_{1 H}\right) \\
0=2 P-\frac{1}{r} \frac{\partial}{\partial r}\left(r \tau_{0 N}\right) \\
\frac{\partial u_{0 N}}{\partial t}=-\frac{1}{r} \frac{\partial}{\partial r}\left(r \tau_{1 N}\right)
\end{gathered}
$$

When $k \leq r \leq \lambda$,

$$
\begin{gathered}
\frac{\partial u_{0 H}}{\partial r}=\left|\tau_{0 H}\right|^{n-1}\left(\left|\tau_{0 H}\right|-n \theta\right) \quad \text { if } \frac{\partial u_{0 H}}{\partial r}>0, \tau_{0 H}<0 \\
\frac{\partial u_{1 H}}{\partial r}=n\left|\tau_{0 H}\right|^{n-2}\left|\tau_{1 H}\right|\left(\left|\tau_{0 H}\right|-(n-1) \theta\right) \quad \text { if } \frac{\partial u_{1 H}}{\partial r}>0, \tau_{1 H}<0 .
\end{gathered}
$$

When $\lambda_{1} \leq r \leq \lambda_{2}$

$$
\begin{array}{cc}
\frac{\partial u_{0 H}}{\partial r}=0 & \text { if }\left|\tau_{0 H}\right|<\theta, \\
\frac{\partial u_{1 H}}{\partial r}=0 & \text { if }\left|\tau_{1 H}\right|<\theta .
\end{array}
$$

When $\lambda_{2} \leq r \leq R_{1}$

$$
\begin{gathered}
\frac{\partial u_{0 H}}{\partial r}=-\left|\tau_{0 H}\right|^{n-1}\left(\left|\tau_{0 H}\right|-n \theta\right) \quad \text { if } \frac{\partial u_{0 H}}{\partial r}<0, \tau_{0 H}>0 \\
\frac{\partial u_{1 H}}{\partial r}=-n\left|\tau_{0 H}\right|^{n-2}\left|\tau_{1 H}\right|\left(\left|\tau_{0 H}\right|-(n-1) \theta\right) \quad \text { if } \frac{\partial u_{1 H}}{\partial r}<0, \tau_{1 H}>0 .
\end{gathered}
$$

When $R_{1} \leq r \leq 1$

$$
\begin{aligned}
& \frac{\partial u_{0 N}}{\partial r}=-\tau_{0 N} \quad \text { if } \frac{\partial u_{0 N}}{\partial r}<0, \tau_{0 N}>0, R_{1} \leq r \leq 1 \\
& \frac{\partial u_{1 N}}{\partial r}=-\tau_{1 N} \quad \text { if } \frac{\partial u_{1 N}}{\partial r}<0, \tau_{1 N}>0, R_{1} \leq r \leq 1
\end{aligned}
$$

The boundary conditions (2.40) become

$$
\begin{gathered}
\frac{\partial u_{0 H}}{\partial r}=\frac{\partial u_{1 H}}{\partial r}=0 \quad \text { at } r=0, \quad u_{0 N}=u_{1 N}=0 \quad \text { at } r=1, \\
u_{0 H}=u_{0 N}, \quad u_{1 H}=u_{1 N}, \quad \tau_{O H}=\tau_{0 N}, \quad \tau_{1 H}=\tau_{1 N} \quad r=R_{1},
\end{gathered}
$$


Equations (2.42)-(2.46) can be solved explicitly with the help of the boundary conditions (2.47). Detailed derivation of the solution to the unknowns variables $u_{0 H}, u_{1 H}, u_{0 N}, u_{1 N}, \tau_{0 H}, \tau_{1 H}, \tau_{0 N}$, and $\tau_{1 N}$ from (2.42)-(2.47) are given by Sankar and Lee [22] and one can go through this reference for the detailed solution. The detailed derivation for the flow rate, wall shear stress, and longitudinal impedance are given by Sankar and Lee [22] and one can go through this reference to know about the details of obtaining these flow quantities.

\section{Results and Discussion}

The objective of the present study is to compare the two-fluid H-B model and two-fluid Casson model. The typical value of the power-law index $n$ of the H-B fluid for blood flow models is generally taken as 0.95 [26]. Though the yield stress of blood at a haematocrit of 40 is $\bar{\tau}_{y}=0.04$ dyne $/ \mathrm{cm}^{2}$ [27], the range $\theta=0$ to 0.1 is more suitable when a catheter is inserted into the blood vessels [3]. Just to pronounce the variations in the flow quantities, we have taken the range of yield stress $\theta$ as 0 to 0.25 in this study. The range $0-0.6$ is used for the catheter radius ratio $k$ [3].

Since the flow is pulsatile and any periodic function can be represented by a Fourier series, it is appropriate to choose the pressure gradient as $P(t)=1+A \sin t$, where $A$ is the amplitude parameter and is taken as less than 1. In the present study, we use the range 0.2-0.5 for the amplitude parameter $A$ to discuss its influence [22]. The ratio $\alpha\left(=\alpha_{N} / \alpha_{H}\right.$ or $\left.\alpha_{N} / \alpha_{C}\right)$ between the Womersley numbers of the Newtonian fluid and H-B fluid or Casson fluid is called Womersley number ratio. It is noted that in this ratio, the numerator corresponds to the Womersley number of the Newtonian fluid and the denominator corresponds to the Womersley number of a nonNewtonian fluid. It is well known that the Womersley number of the Newtonian fluid would be higher than that of the nonNewtonian fluid. Thus, we have chosen the Womersley number ratio as less than 1 and particularly as 0.5. Although the Womersley number $\alpha_{H}$ of the H-B fluid also ranges from 0 to 1 [22], the value 0.5 is used in this study. Given the values of $\alpha$ and $\alpha_{H}$, the value of $\alpha_{N}$ can be obtained from $\alpha=\alpha_{N} / \alpha_{H}$. Similarly, the Womersley number $\alpha_{C}$ of the Casson fluid also ranges from 0 to 1 [23]; the value 0.5 is used in the present study.

\subsection{Yield Plane Locations}

The location of a point where the shear stress is equal to the yield stress is called a yield point and the locus of such points is called yield surface or yield plane. In the case of a tube flow, there is only one yield plane, whereas, for annular flow, there are two yield planes $r=\lambda_{1}$ and $r=\lambda_{2}$ and these two yield planes form the boundary of the plug-flow region. The width of the plug core region is denoted by $\beta$ and is defined as $\beta=\theta / P\left(=\lambda_{2}-\lambda_{1}\right)$, where $\theta$ is the yield stress in the nondimensional form which ranges from $0-0.25, P$ is the nondimensional pressure gradient which is taken as $1+A \sin t$ for pulsatile flow of blood, $A$ is the amplitude of the flow whose range is taken as $0.2-0.5$, and $t$ is the time parameter, Knowing the values of $\theta, A$ and $t$, one can compute the value of $\beta$. For pulsatile flow, the yield plane locations change not only during the course of motion, but also, with respect to the other parameters.

Figure 2 illustrates the width of the plug-flow region of the different fluid models for blood flow through catheterized arteries. The variation of the yield plane locations in a time 


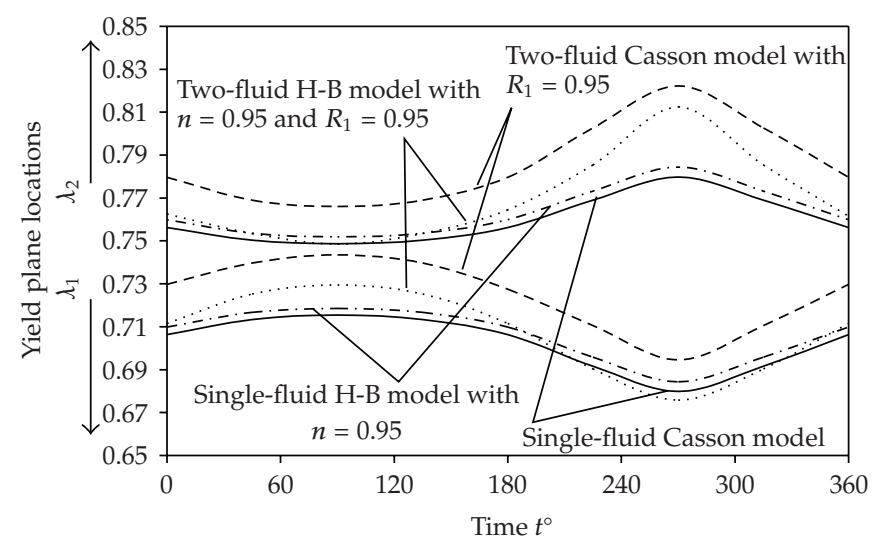

Figure 2: Variation of yield plane locations for different fluid models in a time cycle with $k=A=0.5$ and $\theta=0.05$. The width of the plug-flow region is minimum at $90^{\circ}$ and maximum at $270^{\circ}$. At any instant of time, the width of the plug-flow region of the two-fluid models is marginally lower than that of the single-fluid models and, there is not much difference between the widths of the plug-flow regions of the two-fluid models and the similar behavior is noticed for the single-fluid models.

cycle for different fluid models $k=A=0.5$ and $\theta=0.05$ is depicted in Figure 2. It is clear that for all the fluid models, the width of the plug-flow region decreases as the time parameter $t$ increases from $0^{\circ}$ to $90^{\circ}$ and then it increases as the time variable $t$ increases from $90^{\circ}$ to $270^{\circ}$ and then again it decreases as the time $t$ increases further from $270^{\circ}$ to $360^{\circ}$. The width of the plug-flow region is minimum at $90^{\circ}$ and maximum at $270^{\circ}$. It is also observed that at any instant of time, the width of the plug-flow region of the two-fluid models are marginally lower than those of the single-fluid models. There is not much difference between the widths of the two-fluid H-B model and Casson models and the similar behavior is noticed for the single-fluid H-B and Casson models. It is of importance to note that the plot of the single-fluid H-B model is in good agreement with Figure 2 of Sankar and Hemalatha [7] and the plot of the single-fluid Casson model is in good agreement with Figure 2 of Dash et al. [3].

\subsection{Plug-Flow Velocity}

Figure 3 depicts the simultaneous effects of the nonNewtonian nature of the fluid and the catheter on plug-flow velocity of different two-fluid models for blood flow through catheterized arteries. The variation of the plug-flow velocity with catheter radius ratio $k$ for different two-fluid models with $R_{1}=0.95, \alpha=\alpha_{H}=0.5$ and $A=0.2$ is shown in Figure 3 . The plug-flow velocity for different two-fluid models decreases nonlinearly with the increase of the catheter radius ratio $k$. The plug-flow velocity decreases rapidly as the catheter radius ratio $k$ increases from 0.1 to 0.3 and then it decreases gradually as the catheter radius ratio increases further from 0.3 to 0.6 . It is found that for a given value of the catheter radius ratio $k$, the plug-flow velocity is maximum for the two-fluid power-law model and minimum for the two-fluid Casson model. It is also clear that the plug-flow velocity for the two-fluid H-B model is higher than that of the two-fluid Casson model. It is also observed that the plug-flow velocity decreases slightly with the increase of the power-law index $n$. 


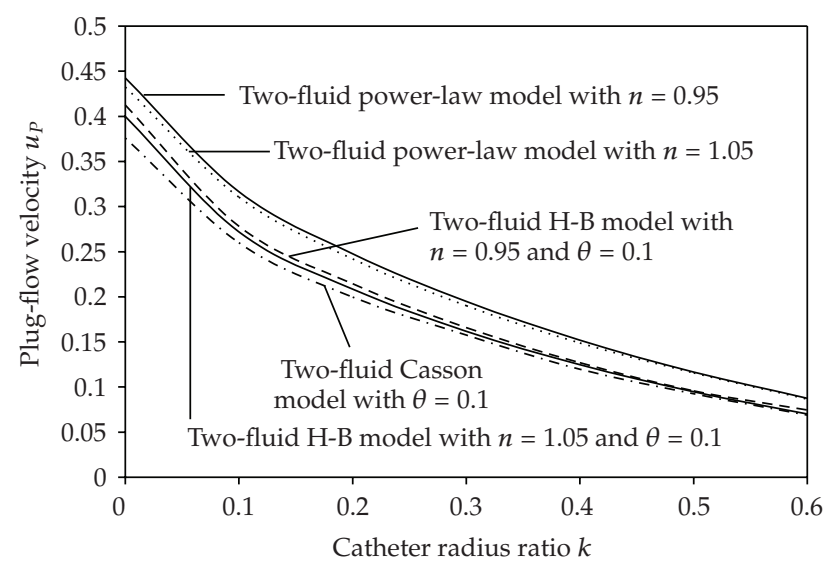

Figure 3: Variation of plug-flow velocity with catheter radius ratio for different fluid models with $R_{1}=0.95$, $\alpha=\alpha_{H}=\alpha_{C}=0.5$ and $A=0.2$. For all the two-fluid models, the plug-flow velocity decreases nonlinearly when the catheter radius ratio $k$ increases from 0.1 to 0.3 and then it decreases linearly when the catheter radius ratio increases further from 0.3 to 0.6 . The plug-flow velocity is maximum for the two-fluid powerlaw model and minimum for the two-fluid Casson model and, the plug-flow velocity for the two-fluid H-B model is higher than that of the two-fluid Casson model. The plug-flow velocity decreases slightly with the increase of the power-law index $n$.

\subsection{Velocity Distribution}

The velocity distribution for different two-fluid models with $k=\alpha=\alpha_{H}=\alpha_{C}=0.5, R_{1}=0.95$ and $A=0.2$ is shown in Figure 4 . One can easily observe the flattened velocity profiles for the two-fluid models, which have fluids with yield stress in the core region, and the usual parabolic velocity profile for the two-fluid power model, which has no yield stress. The twofluid power-law model has the velocity with highest magnitude and the two-fluid Casson model has the velocity with lowest magnitude. The velocity for the two-fluid H-B model is considerably lower than that of the two-fluid power-law model and significantly higher than that of the two-fluid Casson model.

\subsection{Flow Rate}

Figure 5 depicts the transient changes in the flow rate of different fluid models for blood flow through catheterized arteries. The variation of the flow rate in a time cycle for different fluid models with $R_{1}=0.95, A=k=\alpha=\alpha_{H}=\alpha_{C}=0.5$ and $\theta=0.1$ is plotted in Figure 5. It is found that for all the fluid models, the flow rate increases as the parameter $t$ increases from $0^{\circ}$ to $90^{\circ}$ and then it decreases as the time $t$ increases from $90^{\circ}$ to $270^{\circ}$ and then it increases as the time $t$ increases from $270^{\circ}$ to $360^{\circ}$. The flow rate is maximum at $90^{\circ}$ and minimum at $270^{\circ}$. At any instant of time $t$, the flow rate of the two-fluid H-B model is significantly higher than that of the two-fluid Casson model. A similar behavior is observed for the single-fluid H-B and Casson models, but the difference between the flow rates of these models is high when the time parameter $t$ lies between $0^{\circ}$ and $180^{\circ}$ and marginal when the time variable $t$ lies between $180^{\circ}$ and $360^{\circ}$. It is also noticed that for a given set of values of the parameters, the flow rate of the two-fluid H-B model is significantly higher than that of the single-fluid H-B model and the flow rate of the two-fluid Casson model is marginally 


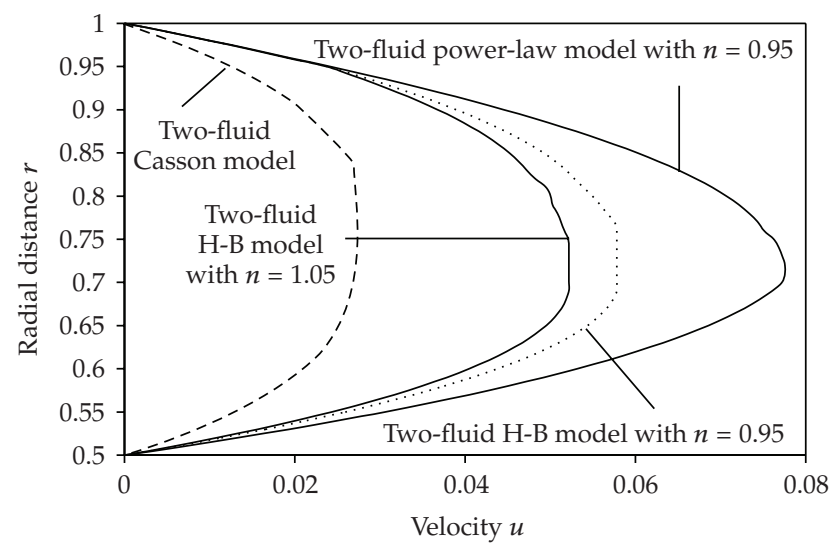

Figure 4: Velocity distribution for different two-fluid models with $k=\alpha=\alpha_{H}=\alpha_{C}=0.5$ and $R_{1}=0.95$ and $A=0.2$. One can note that the flattened velocity profiles for the two-fluid $\mathrm{H}-\mathrm{B}$ and Casson models and the usual parabolic velocity profile for the two-fluid power model. Highest velocity is found for the two-fluid power-law model has the lowest velocity is noticed for the two-fluid Casson. The velocity of the two-fluid H-B model is significantly higher than that of the two-fluid Casson model.

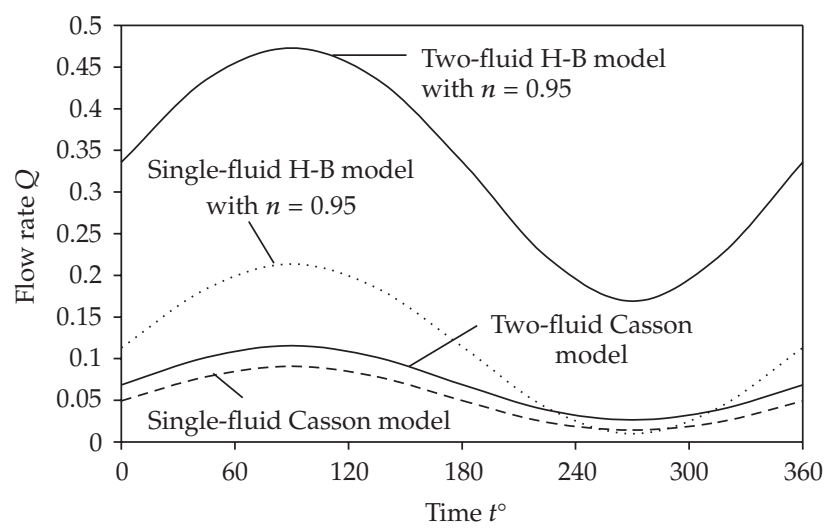

Figure 5: Variation of flow rate in a time cycle for different fluid models with $R_{1}=0.95, t=45^{\circ}, A=k=\alpha=$ $\alpha_{H}=\alpha_{C}=0.5$ and $\theta=0.1$. It is found that for all the fluid models, the flow rate is maximum at $90^{\circ}$ and it is minimum at $270^{\circ}$. At any instant of time $t$, the flow rate of the two-fluid $\mathrm{H}-\mathrm{B}$ model is significantly higher than that of the two-fluid Casson model. A similar behavior is seen for the single-fluid H-B and Casson models. The flow rate of the two-fluid H-B model is significantly higher than that of the single-fluid H-B model.

higher than that of the single-fluid H-B model. It is of interest to note that the plot of the single-fluid H-B model is in good agreement with Figure 7 of Sankar and Hemalatha [7] and the plot of the single-fluid Casson model is in good agreement with Figure 9 of Dash et al. [3].

Figure 6 shows the influence of the nonNewtonian effects on the flow rate of the different two-fluid models. The variation of the flow rate with yield stress for different two-fluid models with $R_{1}=0.95, t=45^{\circ}, k=A=\alpha=\alpha_{H}=\alpha_{C}=0.5$ is depicted in Figure 6. It is observed that the flow rate decreases linearly with the increase of the yield stress $\theta$ for the two-fluid H-B model and it decreases very slowly with the increase of the 


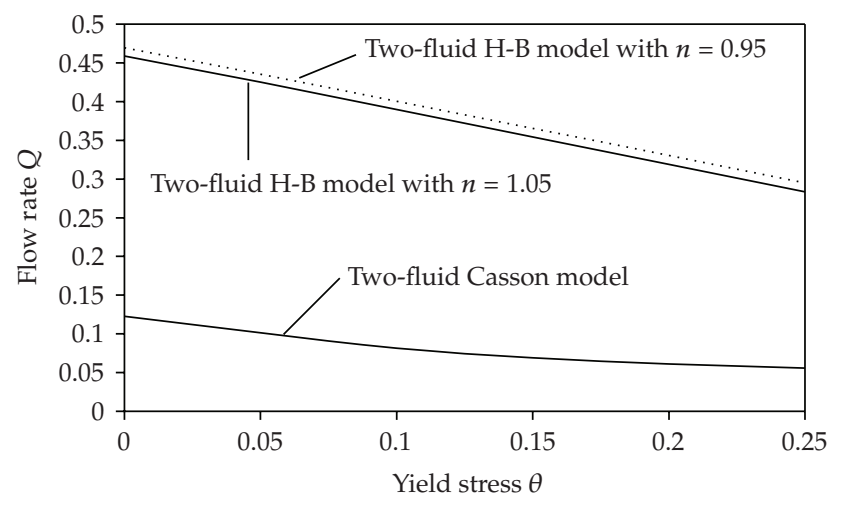

Figure 6: Variation of flow rate with yield stress for different two-fluid models with $R_{1}=0.95, t=45^{\circ}$, $A=k=\alpha=\alpha_{H}=\alpha_{C}=0.5$. It is seen that for increasing values of the yield stress $\theta$, the flow rate decreases linearly for the two-fluid H-B model and it decreases very slowly for the two-fluid Casson model. For a given set of values of the parameters, the flow rate decreases marginally with the increase of the power-law index $n$. The flow rate of the two-fluid H-B model is significantly higher than that of the two-fluid Casson model.

yield stress for the two-fluid Casson model. It is also noted that for a given set of values of the parameters, the flow rate decreases marginally with the increase of the power-law index $n$. One can note that the flow rate of the two fluid H-B model is significantly higher than that of the two-fluid Casson model when all of the other parameters were kept as constant.

\subsection{Wall Shear Stress}

The variation of the wall shear stress in a time cycle for different fluid models with $R_{1}=0.95$, $k=A=\alpha_{N}=0.5$ and $\theta=0.05$ is plotted in Figure 7. It is observed that the wall shear stress increases when the time parameter $t$ increases from $0^{\circ}$ to $90^{\circ}$ and then it decreases as the time $t$ increases from $90^{\circ}$ to $270^{\circ}$ and then it increases when the time $t$ increases from $270^{\circ}$ to $360^{\circ}$. The wall shear stress is maximum at $90^{\circ}$ and minimum at $270^{\circ}$. It is found that for a given set of values of the parameters, the wall shear stress of the two-fluid models are marginally lower than those of the single-fluid models. Also, it is noticed that the wall shear stress of the two-fluid H-B model is marginally lower than that of the two-fluid Casson model. It is of interest to note that the plot of the single-fluid H-B model is in good agreement with Figure 9 of Sankar and Hemalatha [7] and the plot of the single-fluid Casson model is in good agreement with Figure 11 of Dash et al. [3].

Figure 8 shows the effects of catheterization on wall shear stress of the different fluid models for blood flow through catheterized arteries. The variation of wall shear stress with catheter radius ratio for different fluid models with $R_{1}=0.95, t=45^{\circ}, \theta=0.05$ and $A=\alpha_{N}=$ 0.5 is sketched in Figure 8. It is seen that the wall shear stress decreases almost linearly with the increase of the catheter radius ratio for all the fluid models. For a given set of values of the parameters, the wall shear stress of the two-fluid models is slightly higher than that of the single-fluid models. There is not much of the difference between the wall shear stress of two-fluid H-B and Casson models. 


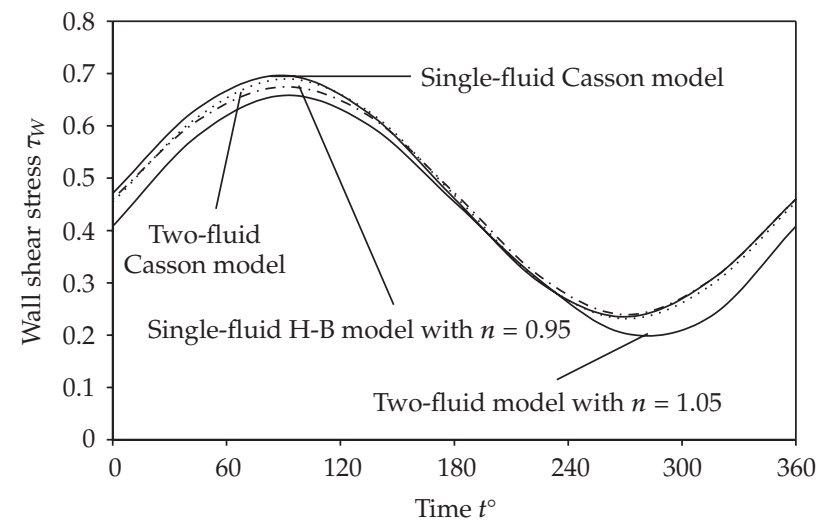

Figure 7: Variation of wall shear stress in a time cycle for different fluid models with $R_{1}=0.95, A=k=$ $\alpha_{N}=0.5$ and $\theta=0.05$. It is observed that the wall shear stress increases when the time parameter $t$ increases from $0^{\circ}$ to $90^{\circ}$ and then it decreases as the time $t$ increases from $90^{\circ}$ to $270^{\circ}$ and then it increases when the time $t$ increases from $270^{\circ}$ to $360^{\circ}$. For a given set of values of the parameters, the wall shear stress of the two-fluid models are marginally lower than those of the single-fluid models and the wall shear stress of the two-fluid H-B model is marginally lower than that of the two-fluid Casson model.

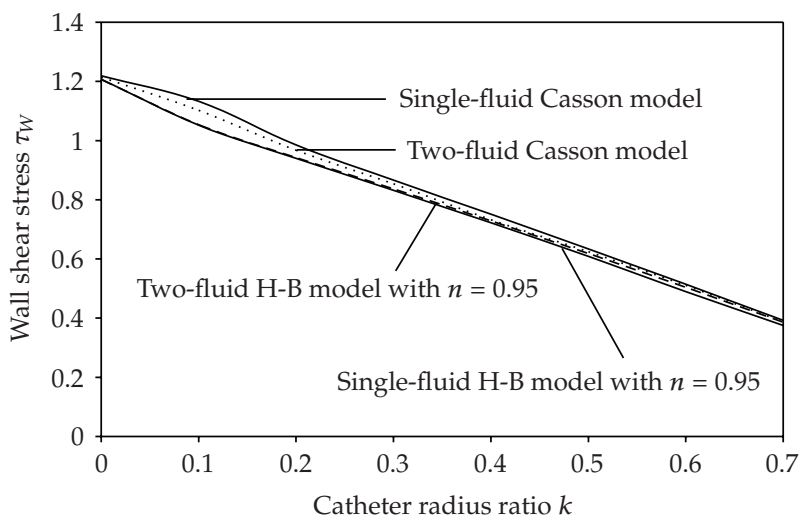

Figure 8: Variation of wall shear stress with catheter radius ration different fluid models with $R_{1}=0.95$, $t=45^{\circ}, \theta=0.1$ and $A=\alpha_{N}=0.5$. One can observe that for all the fluid models, the wall shear stress decreases almost linearly with the increase of the catheter radius ratio. For a given set of values of the parameters, the wall shear stress of the two-fluid models is slightly higher than that of the single-fluid models. There is only a slight difference between the wall shear stresses of two-fluid H-B and Casson models. This graph exhibits the effects of catheterization on wall shear stress of the different fluid models for blood flow through catheterized arteries.

\subsection{Resistance to Flow}

Figure 9 shows the variation of the longitudinal impedance to flow with yield stress $\theta$ for twofluid H-B and Casson models with $k=A=\alpha=\alpha_{H}=\alpha_{C}=0.5$ and $t=45^{\circ}$. It is observed that for the two-fluid H-B model, the longitudinal impedance to flow increases very slowly with the increase of the yield stress, but, for the two-fluid Casson model, the impedance increases linearly when the yield stress increases from 0 to 0.15 and then it increases slowly when the yield stress increases from 0.15 to 0.25 . The longitudinal impedance to flow of the two-fluid $\mathrm{H}-\mathrm{B}$ model is significantly lower than that of the two-fluid Casson model. 
Table 1: Estimates of the increase in the longitudinal impedance for two-fluid H-B model and two-fluid Casson model with effects on the catheterization and yield stress with $R_{1}=0.95, A=\alpha=\alpha_{H}=\alpha_{C}=0.5$, and $t=45^{\circ}$.

\begin{tabular}{lcccccccc}
\hline Catheter radius ratio $k$ & \multicolumn{3}{c}{ Two-fluid H-B model with $n=0.95$} & \multicolumn{4}{c}{ Two-fluid Casson model } \\
& $\theta=0.1$ & $\theta=0.15$ & $\theta=0.2$ & $\theta=0.25$ & $\theta=0.1$ & $\theta=0.15$ & $\theta=0.2$ & $\theta=0.25$ \\
\hline 0.1 & 1.336 & 1.353 & 1.372 & 1.392 & 1.423 & 1.439 & 1.450 & 1.458 \\
0.2 & 1.714 & 1.752 & 1.793 & 1.839 & 1.994 & 2.029 & 2.055 & 2.069 \\
0.3 & 2.243 & 2.315 & 2.397 & 2.491 & 2.892 & 2.959 & 3.004 & 3.022 \\
0.4 & 3.023 & 3.168 & 3.329 & 3.519 & 4.456 & 4.577 & 4.642 & 4.652 \\
0.5 & 4.257 & 4.529 & 4.857 & 5.262 & 7.478 & 7.679 & 7.753 & 7.864 \\
\hline
\end{tabular}

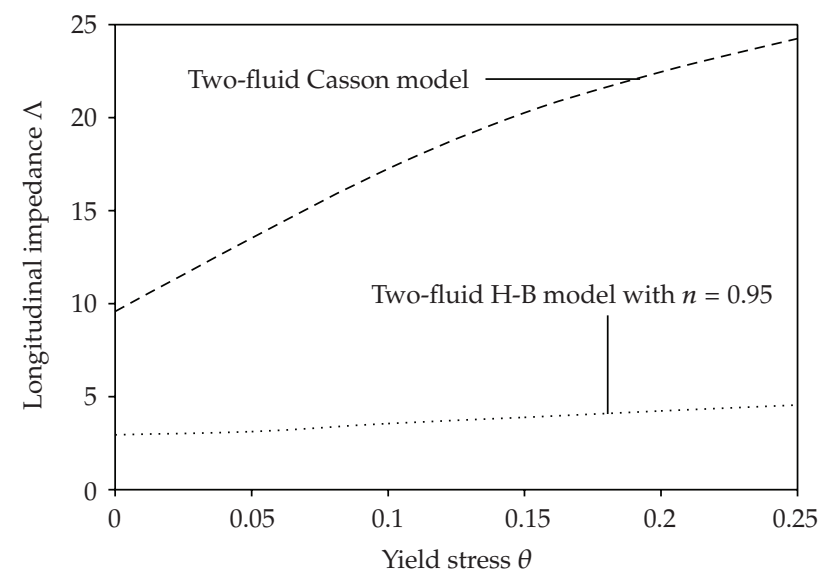

Figure 9: Variation of longitudinal impedance with yield stress for two-fluid H-B and Casson models with $A=k=\alpha=\alpha_{H}=\alpha_{C}=0.5, R_{1}=0.95$ and $t=45^{\circ}$. When the yield stress increases, the longitudinal impedance to flow increases very slowly two-fluid H-B model and it increases linearly for the two-fluid Casson model when the yield stress increases from 0 to 0.15 and then it increases slowly when the yield stress increases from 0.15 to 0.25 . The longitudinal impedance to flow of the two-fluid H-B model is significantly lower than that of the two-fluid Casson model.

The increase in the longitudinal impedance due to the catheterization is defined as the ratio between the longitudinal impedance of a fluid model in a catheterized artery for a given set of values of the parameters and the longitudinal impedance of the same fluid in the uncatheterized artery for the same set of values of the parameters [23]. The estimates of the increase in the longitudinal impedance with effects on catheterization for different values of the yield stress $\theta$ for the two-fluid H-B and Casson models with $R_{1}=0.95, k=A=\alpha=\alpha_{H}=$ $\alpha_{C}=0.5$ and $t=45^{\circ}$ are given in Table 1 . For the range $0.1-0.5$ of the catheter radius ratio, the range of increase in longitudinal impedance of the two-fluid H-B model are 1.34-4.26, $1.35-4.53,1.37-4.86$ and $1.39-5.26$ when the yield stress $\theta$ values are $0.1,0.15,0.2$ and 0.25 , respectively. For the two-fluid Casson model, the estimates of the increase in the longitudinal impedance increase are $1.42-7.47,1.44-7.68,1.45-7.75$ and $1.46-7.86$ when the yield stress $\theta$ values are $0.1,0.15,0.2$ and 0.25 , respectively. It is important to note that the estimates of the increase in the longitudinal impedance considerably much smaller for the two-fluid H-B model than those of the two-fluid Casson model.

Catheters play an important role in the clinical investigations, since they are used to measure different types of flow quantities. Some types of catheters used in clinics, their sizes 
Table 2: Different types of catheters used in cardiovascular treatment, their sizes, and the flow quantities measured using them.

\begin{tabular}{lcl}
\hline Type of catheter & Catheter diameter $d_{i}(\mathrm{~mm})$ & Flow quantity measured \\
\hline Angioplasty catheter guidewire & 0.356 & Pressure drop \\
Coronary angioplasty catheter & 1.400 & Pressure distal to lesion \\
Guiding catheter & 2.600 & Pressure at coronary ostium \\
Doppler catheter & 1.000 & Velocity proximal to lesion \\
Coronary infusion catheter & 0.660 & Pressure drop across lesion \\
\hline
\end{tabular}

Table 3: Range of increase in the longitudinal impedance for different types of catheters for two-fluid H-B model and two-fluid Casson model with $A=\alpha=\alpha_{H}=\alpha_{C}=0.5, R_{1}=0.95, t=45^{\circ}$, and $\theta=0.1$.

\begin{tabular}{lccc}
\hline Type of catheter & $\begin{array}{c}\text { Range of catheter } \\
\text { size } d_{i} / d_{0}\end{array}$ & $\begin{array}{c}\text { Two-fluid H-B } \\
\text { model with } n=0.95\end{array}$ & $\begin{array}{c}\text { Two-fluid } \\
\text { Casson model }\end{array}$ \\
\hline Guidewire & $0.08-0.18$ & $1.143-1.303$ & $1.159-1.375$ \\
Infusion & $0.14-0.33$ & $1.239-1.569$ & $1.264-1.768$ \\
Angioplasty catheter & $0.3-0.6$ & $1.512-2.314$ & $1.641-3.558$ \\
\hline
\end{tabular}

and their usage are mentioned in Table 2 [20], where $d_{i}$ is the diameter of the catheter and $d_{0}$ is the diameter of the artery. As an application of the present study to the medical field, the different types of the catheters (with sizes), which are used in the medical field [23], and the corresponding range of estimates of the increase in the longitudinal impedance for the two-fluid H-B and Casson models with $\theta=0.1, k=A=\alpha=\alpha_{H}=\alpha_{C}=0.5$ and $t=45^{\circ}$ and $R_{1}=0.95$ are computed in Table 3 . It is observed that the range of estimates of the increase in the longitudinal impedance to flow for the two-fluid H-B model are significantly very lower than those of the two-fluid Casson model. Hence, it is strongly felt that the twofluid H-B model will have more applicability than the two-fluid Casson model in the clinical use.

\subsection{Usefulness of the Present Study}

Figure 4 could be useful to the physicians in predicting the postcatheterization velocity profiles and thus, they can predict the effect of introducing the catheter on the velocity profiles and flow rate of the blood in the artery. Figure 8 might be useful to clinicians to predict and analyze the wall shear stress after inserting the catheter into the artery coaxially. Tables 1 and 3 might be used by clinicians to obtain the rough estimates of increase in longitudinal impedance due to the insertion of the catheter into the artery and the influence of nonNewtonian behavior of blood on impedance to flow. Since, catheters are used widely clinically; these estimates might be useful to physicians to decide their future course of action. Furthermore, as catheters are used clear the short occlusions or stenosis in the arterial wall, the present study could also be useful in estimating the increase in the longitudinal impedance and wall shear stress, since, the insertion of a catheter into the artery alters the flow field, modifies the pressure distribution and hence increases the flow resistance. Thus, there is considerable usefulness of the present study in the physiological context. 


\section{Conclusions}

The pulsatile flow of blood through catheterized arteries is analyzed, assuming blood as a (i) two-fluid Casson model and (ii) two-fluid Herschel-Bulkley model. This study brings out the advantages of using the two-fluid Herschel-Bulkley (H-B) model over the two-fluid Casson model for pulsatile blood flow through catheterized arteries. The effects of the catheterization, nonNewtonian nature of blood and pulsatility of the flow on the yield plane locations, velocity, flow rate, wall shear stress and longitudinal impedance are analyzed for different two-fluid models. It is found that the width of the plug-flow velocity, velocity distribution and thee flow rate for the two-fluid H-B model are considerably higher than those of the twofluid Casson model for a given set of values of the parameters. Also, it is observed that the longitudinal impedance is significantly very low for the two-fluid H-B model than those of the two-fluid Casson model. It is of interest to note that the difference between the estimates of the increase in the longitudinal impedance of the two-fluid H-B model and the two-fluid Casson model is substantial and hence, one can expect a significant increase in the flow of the two-fluid H-B model. Thus, it is concluded that the two-fluid H-B model will have more applicability in analyzing the blood flow through catheterized arteries.

\section{References}

[1] A. Sarkar and G. Jayaraman, "Correction to flow rate-pressure drop relation in coronary angioplasty: steady streaming effect," Journal of Biomechanics, vol. 31, pp. 781-791, 1998.

[2] G. Jayaraman and K. Tiwari, "Flow in a catheterized curved artery," Medical and Biological Engineering and Computing, vol. 33, pp. 1-6, 1995.

[3] R. K. Dash, G. Jayaraman, and K. N. Metha, "Estimation of increased flow resistance in a narrow catheterized artery-a theoretical model," Journal of Biomechanics, vol. 29, pp. 917-930, 1996.

[4] R. V. Anderson, G. S. Roubin, P. P. Leimgruber, et al., "Measurement of trans-stenotic pressure gradient during percutaneous transluminal coronary angioplasty," Circulation, vol. 73, pp. 1223-1230, 1986.

[5] R. Wilson, M. R. Johnson, M. L. Marcus, et al., "The effect of coronary angioplasty on coronary flow reserve," Circulation, vol. 77, pp. 873-885, 1988.

[6] R. K. Dash, G. Jayaraman, and K. N. Metha, "Flow in a catheterized curved artery with stenosis," Journal of Biomechanics, vol. 32, pp. 49-61, 1999.

[7] D. S. Sankar and K. Hemalatha, "Pulsatile flow of Herschel-Bulkley fluid through catheterized arteries—a mathematical model," Applied Mathematical Modeling, vol. 31, pp. 1497-1517, 2007.

[8] L. H. Back, E. Y. Kwack, and M. R. Back, "Flow rate-pressure drop relation in coronary angioplasty: catheter obstruction effect," Journal of Biomechanical Engineering, vol. 118, pp. 83-89, 1996.

[9] H. Kanai, E. Y. Kwack, and M. R. Back, "Flow rate-pressure drop relation in coronary angioplasty: catheter obstruction effect," Journal of Biomechanical Engineering, vol. 118, pp. 83-89, 1996.

[10] T. A. MacMahon, C. Clark, V. S. Murthy, and A. H. Shapiro, "Intraaortic balloon experiment in a lumped-element hydraulic model of the circulation," Journal of Biomechanics, vol. 4, pp. 335-350, 1971.

[11] K. V. Manjula and R. Devanathan, "A theoretical study of catheter probe in a stenosed artery," in Biomechanics, K. B. Sahay and R. K. Saxena, Eds., pp. 240-246, Wiley Eastern, New Delhi, India, 1988.

[12] L. H. Back, "Estimated mean flow resistance increase during coronary artery catheterization," Journal of Biomechanics, vol. 27, pp. 169-175, 1994.

[13] K. T. Karahalios, "Some possible effects of a catheter on the arterial wall," Medical Physics, vol. 17, pp. 922-925, 1990.

[14] G. Jayaraman and R. K. Dash, "Numerical study of flow in a constricted curved annulus: an application to flow in a catheterised artery," Journal of Engineering Mathematics, vol. 40, no. 4, pp. 355-376, 2001.

[15] D. A. MacDonald, "Pulsatile flow in a catheterized artery," Journal of Biomechanics, vol. 19, pp. 239-249, 1986.

[16] R. K. Dash and P. Daripa, "Analytical and numerical studies of a singularly perturbed Boussinesq equation," Applied Mathematics and Computation, vol. 126, no. 1, pp. 1-30, 2002. 
[17] C. Tu and M. Deville, "Pulsatile flow of non-Newtonian fluids through arterial stenosis," Journal of Biomechanics, vol. 29, pp. 899-908, 1996.

[18] P. Chaturani and R. P. Samy, "Pulsatile flow of a Casson fluid through stenosed arteries with application to blood flow," Biorheology, vol. 23, no. 5, pp. 499-511, 1986.

[19] V. P. Srivastava and M. Saxena, "Two-layered model of Casson fluid flow through stenotic blood vessels: applications to the cardiovascular system," Journal of Biomechanics, vol. 27, pp. 921-928, 1994.

[20] D. S. Sankar and K. Hemalatha, "A non-Newtonian fluid flow model for blood flow through a catheterized artery—steady flow," Applied Mathematical Modeling, vol. 31, pp. 1847-1864, 2007.

[21] J. C. Misra and S. K. Pandey, "Peristaltic transport of blood in small vessels: study of a mathematical model," Computers E Mathematics with Applications, vol. 43, no. 8-9, pp. 1183-1193, 2002.

[22] D. S. Sankar and U. Lee, "Two-fluid nonlinear mathematical model for pulsatile blood flow through catheterized arteries," Journal of Mechanical Science and Technology, vol. 23, pp. 1650-1669, 2009.

[23] D. S. Sankar, "A two-fluid model for pulsatile flow in catheterized blood vessels," International Journal of Non-Linear Mechanics, vol. 44, pp. 337-351, 2009.

[24] P. Chaturani and R. P. Samy, "A study of non-Newtonian aspects of blood flow through stenosed arteries and its applications in arterial diseases," Biorheology, vol. 22, pp. 521-531, 1985.

[25] N. Iida, "Influence of plasma layer on steady blood flow in microvessels," Japanese Journal of Applied Physics, vol. 17, pp. 203-214, 1978.

[26] D. S. Sankar and U. Lee, "Two-fluid non-linear model for flow in catheterized blood vessels," International Journal of Non-Linear Mechanics, vol. 43, pp. 622-631, 2008.

[27] E. W. Merrill, "Rheology of blood," Physiological Reviews, vol. 49, pp. 863-888, 1969. 


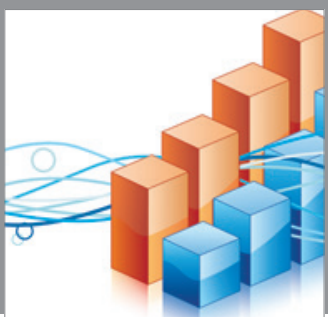

Advances in

Operations Research

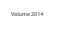

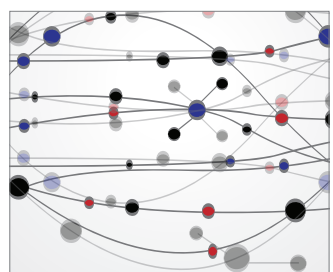

\section{The Scientific} World Journal
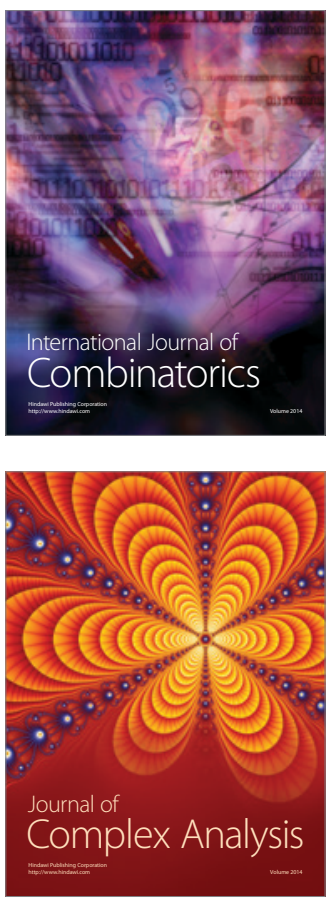

International Journal of

Mathematics and

Mathematical

Sciences
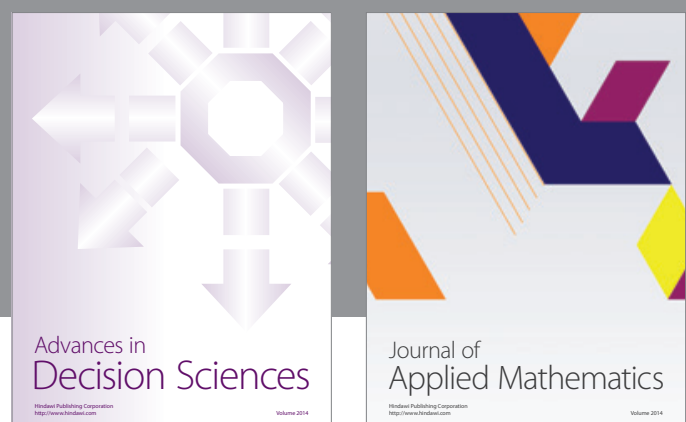

Journal of

Applied Mathematics
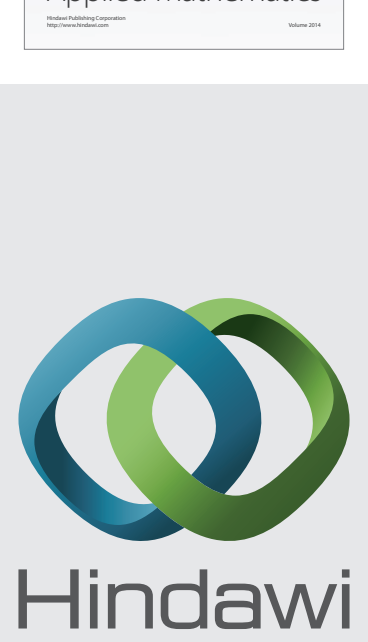

Submit your manuscripts at http://www.hindawi.com
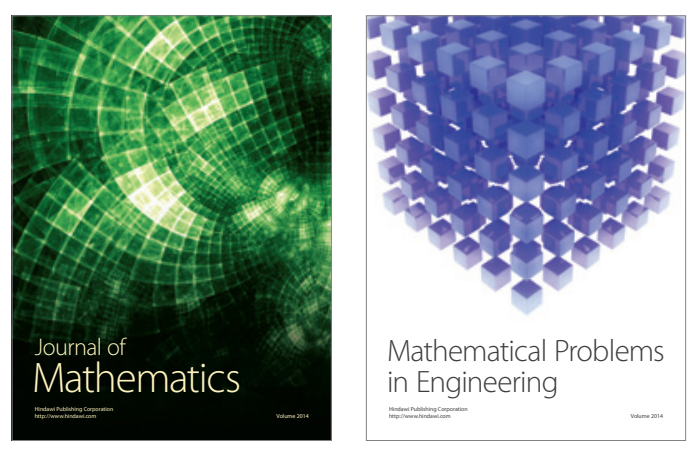

Mathematical Problems in Engineering
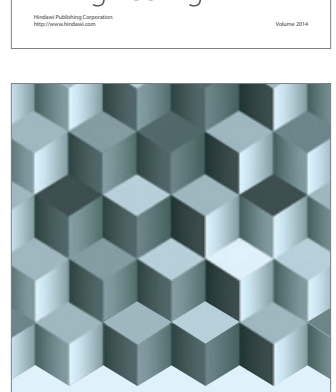

Journal of

Function Spaces
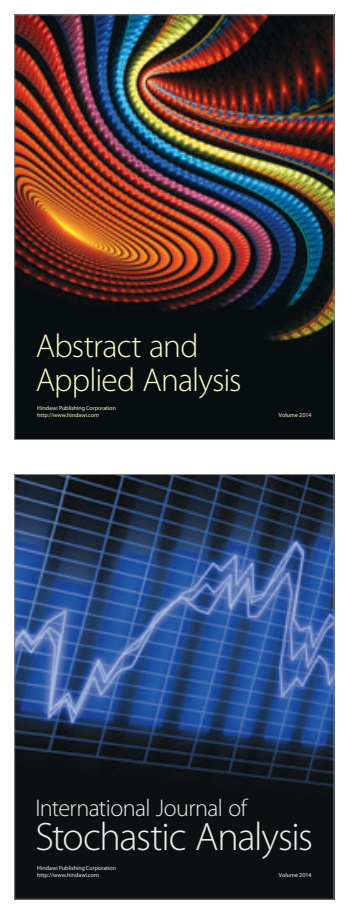

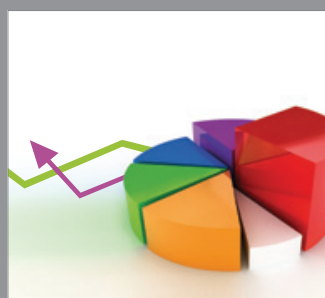

ournal of

Probability and Statistics

Promensencen
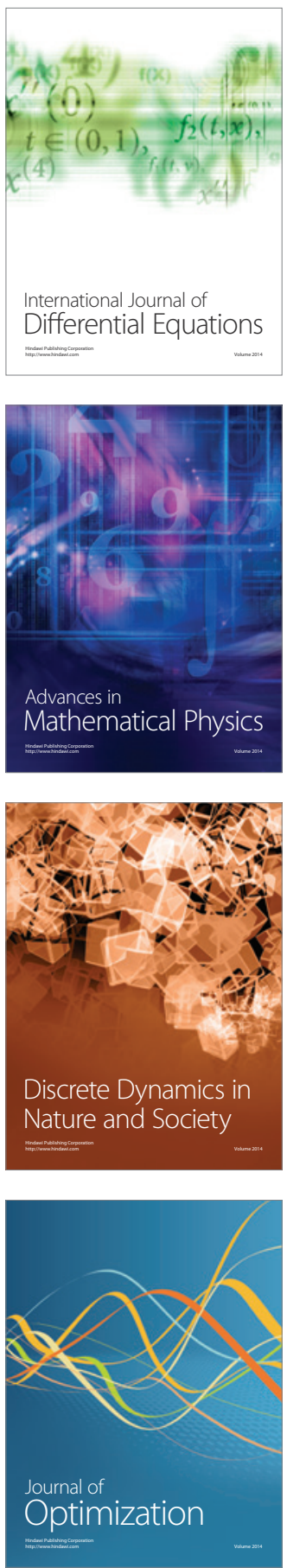